\title{
Téoros
}

Revue de recherche en tourisme

\section{Guides touristiques, racontez-nous des histoires...}

\section{Marc Laplante et Louise Trottier}

Volume 4, numéro 1, mars 1985

L'information touristique

URI : https://id.erudit.org/iderudit/1080743ar

DOI : https://doi.org/10.7202/1080743ar

Aller au sommaire du numéro

Éditeur(s)

Université du Québec à Montréal

ISSN

0712-8657 (imprimé)

1923-2705 (numérique)

Découvrir la revue

Citer cet article

Laplante, M. \& Trottier, L. (1985). Guides touristiques, racontez-nous des histoires... Téoros, 4(1), 22-24. https://doi.org/10.7202/1080743ar d'utilisation que vous pouvez consulter en ligne.

https://apropos.erudit.org/fr/usagers/politique-dutilisation/ 


\title{
Guides touristiques, racontez-nous des histoires...
}

\author{
par Marc Laplante et Louise Trottier*
}

Qu'est-ce qu'un guide touristique? Un instrument d'initiation à la "dominance", comme l'affirmait M. B. Lerivray, en 1975, dans son livre au titre séducteur: Guides bleus, guides verts et lunettes roses ${ }^{(1 /}$ ? Parce que les guides Michelin vous recommandent fréquemment de monter à des sommets, de vous arrêtter à des belvedères, de grimper des centaines de marches pour atteindre le haut des tours ou des clochers, faut-il voir là une "pédagogie" de la classe dominante?

Un guide est-il plutót un instrument d'information? Oui, peut-êtrel Mais il fait des choix, favorise des lieux, "oublie" bien des choses, n'est pas à jour, exagère souvent la valeur de tel ou tels événements ou monuments, etc. Alors, peut-être est-il un outil de promotion, de propagande? Com= ment distinguer alors le guide touristique des dépliants, brochures, encarts publicitaires, des affiches, panneaux, messages audio-visuels? Les tenants de l'information et de la promotion savent que la frontiẻre entre leur domaine est molle et poreuse. S'ils le veulent, ils peuvent se renvoyer la balle fort longtemps.

Obligés d'aborder l'étude des fonctions de ces guides touristiques dans le cadre d'une recherche toute récente sur la méthodologie d'evaluation des attractions touristiques ${ }^{\mid 2\}}$, nous avons dû déborder ces idées répandues et trouver autre chose. Brièvement, en quelques paragraphes, nous situerons d'abord les guides touristiques dans l'expérience touristique globale, dans leurs rapports avec le touriste d'une part et les attractions d'autre part, et nous présenterons ensuite quelques résultats de nos premiẻres applications sur le terrain. dans la région de Québec. La recherche n'étant pas terminée, nous serons prudents dans l'interprétation des résultats et nous prions le lecteur de recevoir nos "conclusions" comme des connaissances très provisoires.

\footnotetext{
"Marc Laplante est professeur en tourisme â I'UOAM
} et Louise Trottier est documentaliste.

\section{Les guides touristiques et leurs étoiles...}

Le projet de recherche dont il s'agit ici est né d'une demande des services d'accueil et de renseignements touristiques du minisment à plusieurs grands guides touristiques célébres, les nôttres au Québec ne présentent pas les attractions touristiques accompagnées d'étoiles marquant leur importance (on le fait pour les chambres et les repas seulement). Comment développer ici un tel système d'évaluation? Et d'abord, comment les auteurs de ces grands guides procédent-ils pour arriver à mettre 3,4 ou 5 étoiles à un panorama, à un monument historique ou à une fête au village?

Interroger directement les auteurs de ces guides à ce propos revenait ả demander à un entrepreneur de vous livrer les secrets de sa réussite. Chercher une littérature scientifique pouvant vous éclairer à ce propos n'a rien donné; multiplier les consultations de travaux comme celui de Lerivray cité plus haut risquait de nous garder dans cette seule optique. Alors, il fallait procé der nous-mêmes à l'enquète, tenter d'établir des critères d'évaluation des attractions touristiques, puis construire des mesures, tère du Tourisme du Québec ${ }^{13}$. Contraire-

les appliquer et ordonner ainsi hiérarchiquement les attractions d'un territoire les unes par rapport aux autres. Projet utopique, à première vue, qui nous a conduits toutefois à la recherche thérique et à l'analyse conceptuelle intensive.

\section{Pourquoi n'y a-t-il d'attractions que touristiques?}

Le concept d'attraction touristique, quand on $y$ pense un moment, est très intriguant. II est employé quasi universellement et depuis un siècle pour désigner ce qui attire les touristes. Curieusement, on ne parle pas d'attractions écologiques pour dire ce qui attire les amateurs de plein air; on ne dit pas: attractions urbaines pour référer à ces choses qui font le charme et l'attrait des grandes villes, etc. Non! Seules les choses offertes aux touristes à titre de "produits" portent le nom d'attractions! On est tenté de croire alors qu'il faut en mettre de la pression pour décider un touriste à partir, qu'il faut sortir les mots les plus séduisants pour l'intéresser, etc. On est surtout amené à s'interroger, une fois de plus, sur le touriste et sur les relations qui doivent exister entre celui-ci et les attractions désignées nommément comme "touristiques".

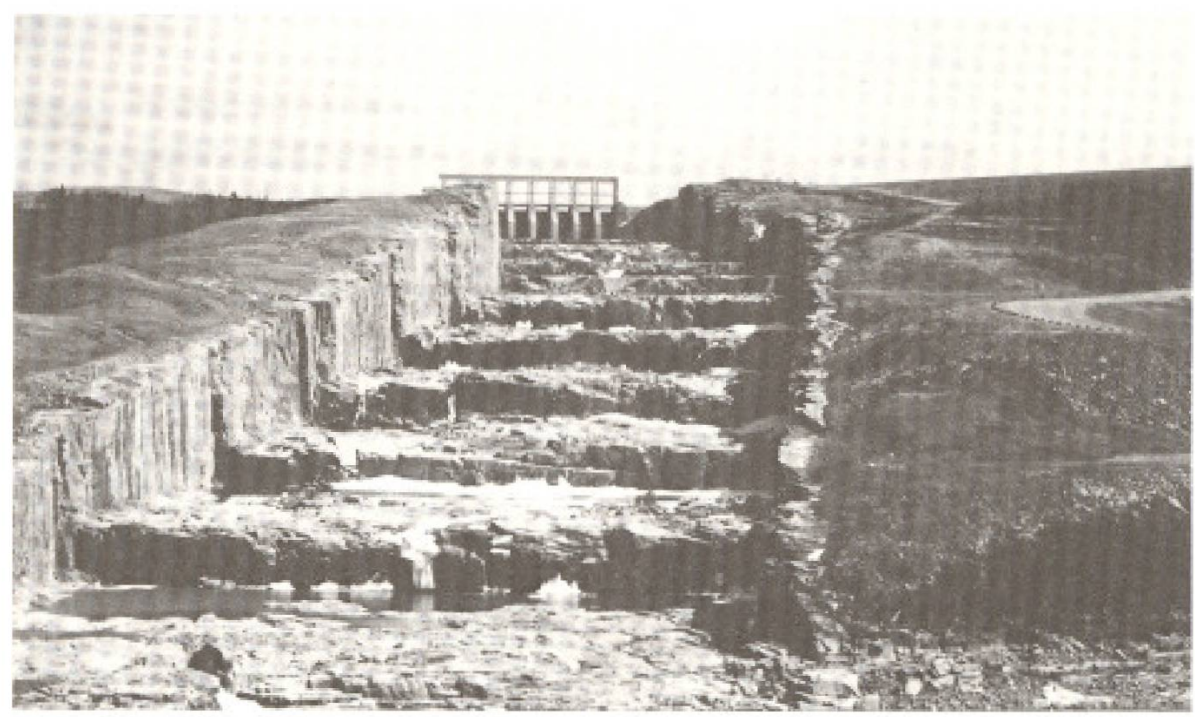

Pour qu'une ressource, si riche soit-elle en elle-mẻme, devienne touristique, il faut la transformer en une attraction. Comment? Sur la photo, l'évacuateur de crues à LG-2. (Baie James) 
Tel fut notre cheminement. II nous a conduit à reprendre d'abord et à appliquer ensuite, le cadre théorique de Dean MacCannell que nous avions brièvement exposé dans un numéro de Téoros, paru à l'été $1983^{|4|}$.

\section{Une ressource touristique devient attraction par la sacralisation}

MacCannell définit d'abord l'attraction comme une relation de fait (empirique) entre un touriste, une chose-â-voir (un sight) et un élément d'information à propos de ce sight (un marker). Touristes et sights "communiquent" par le canal des markers. Donc, impossible de considérer séparément le touriste ou le sight. II n'y a pas d'attractions "naturelles" ou toutes faites. Par contre, tout, absolument tout dans une société peut devenir une attraction touristique, si on l'accompagne de bons markers.

On parle couramment de ressources touristiques (naturelles, culturelles, etc.) pour désigner les richesses d'ici qui peuvent intéresser les touristes. Mais il n'y a rien d'intrinsèquement touristique dans ces ressources.

Pour qu'une ressource, si riche soit-elle en elle-même, devienne touristique, il faut la transformer en une attraction. Comment? Par un processus que MacCannell appelle SACRALISATION.

La religion a toujours su "investir de sacré" ses lieux de cultes, ses cérémonies, etc. Le tout prend l'allure d'un spectacle, souvent très impressionnant. Les participants s'intègrent à ces événements en suivant les rituels imposés par l'observation assidue des comportements les plus fréquents des touristes (classe movenne internationale). MacCannell a retrouvé, sous forme très laīcisée, les rituels et les "mises-en-scène" du temps des ferveurs religieuses populaires. La sacralisation désigne donc tout ce qui est fait pour rendre attirant, fascinant, séduisant un lieu, un événement, une région, etc. Toute la durée du voyage peut se concevoir comme une longue "représentation" - genre happening - avec des temps forts, des pauses, des moments de gaieté, de grandes émotions, etc.

\section{Les guides touristiques sont des éléments du processus de sacralisation}

Pour faire bref maintenant, disons que la sacralisation se fait á la fois "on-sight" et "off-sight", sur les lieux même de l'attraction et à l'extérieur. La sacralisation offsight opère principalement (mais non uniquement) aै travers les guides touristiques. Un guide, agissant ainsi avant l'arrivée sur les lieux, a pour première fonction de rendre crédible la promesse de bonheur (appelée aussi, selon le cas, aventure, plaisir, repos, fun, distraction, évasion, rencontres, découvertes, etc.) que le touriste achète en faisant ses réservations. Comme il n'achète rien d'autre que cela, comme il investit, pour ce plaisir, un temps rare (celui des vacances) et un budget précieux, le touriste ne veut pas revenir déçu de son voyage. La sacralisation, sous tous ces aspects, sert donc à assurer que les promesses de bonheur seront tenues.

\section{Évaluer les sacralisations}

Les guides touristiques peuvent être analysés en tant que soutien à la sacralisation. Leur discours est, avant tout, une contribution à la sacralisation des ressources. Pratiquement, on peut faire l'analyse de contenu d'un guide touristique pour évaluer comment il contribue à sacraliser chacune des ressources qu'il présente. Des recherches sur le vocabulaire des guides seraient intéressantes mais complexes. Nous avons préféré, en un premier temps, construire une mesure plus simple.

L'index-guide, élaboré pour l'expérimentation dans la région de Québec, comptait sept indicateurs pondérés de la façon indiquée au tableau 1.

Neuf livres-guides ont été entièrement dépouillés. Un dossier était constitué pour chaque attraction. $\mathrm{C}^{i}$ est ainsi que chaque texte tiré d'un guide pour une attraction donnée recevait un score. La cumulation des évaluations donnait un score total â la fin du dépouillement de livres-guides. Sur les guides ainsi dépouillés, cinq étaient régionaux ou locaux, un était national et trois, internationaux ${ }^{(5)}$. Une attraction, présente dans des guides nationaux et internationaux, recevait un coefficient multiplicateur, après son évaluation à partir des sept indicateurs décrits ci-haut (un guide international est forcé de faire des choix; ce qu'il retient doit être très "fort").

Le score total que recevait une attraction à la fin du dépouillement des guides était ramené sur 100 . Finalement, les scores ont été répartis en quatre classes: A: attraction très sacralisée, B: assez sacralisée, $C$ : peu sacralisée et D: très peu sacralisée là peine mentionnée).

La liste initiale comptait 504 attractions. L'équipe de recherche, en a évalué sur place (on signt) 305 (plusieurs furent regroupées). L'évaluation de cette sacralisation sur le terrain (on sight) consistait à identifier et à juger de la qualité des markers qui étaient associés à la ressource pour la rendre intéressante aux visiteurs (spectacles audio-visuels dans certaines maisons de la Place-Royale, écriteaux descriptifs, tables d'orientation, maquettes, etc.). La qualité de la sacralisation sur le terrain s'est exprimée finalement par un index en quatre niveaux $(A, B, C, D)$ comme pour l'index-guide. (Voir tableau 2)

\section{Tableau 1: \\ Pondération des indicateurs}

1. Longueur du texte consacré à l'attraction dans le guide (en fraction de page)

2. Photo accompagnant la description de l'attraction: oui ou non 0

3. Dessin accompagnant la description: oui-non $\quad 0 \quad 10$

4. L'attraction est indiquée sur une carte? oui-non $\quad 0 \quad 10$

5. L'attraction est nommée sur un circuit? oui-non $\quad 0 \quad 10$

6. L'attraction sert d'identificateur lex.: sur la page couverture) oui-non $\quad \begin{array}{lll}0 & 20\end{array}$

7. L'attraction fait partie d'un ensemble et en est l'élément majeur? Non $=0$, Oui $=5$; oui, élément majeur $=15$

Tableau 2:

Comparaison de deux scores de sacralisation Région de Québec-Portneuf, été 1985

\section{Classes}

A (très sacralisé)

B (assez sacralisé)

C (peu sacralisé)

D (très peu sacralisé)

\begin{tabular}{cr} 
Scores & Scores \\
Guides & Terrain \\
$20 \%$ & $5 \%$ \\
$11 \%$ & $8 \%$ \\
$43 \%$ & $43 \%$ \\
$26 \%$ & $44 \%$ \\
\hline $100 \%$ & $100 \%$
\end{tabular}




\section{Promesses et réalités}

Comparant les scores-guides aux scoresterrains, nous avons constaté que les premiers étaient plus forts que les seconds dans $74 \%$ des cas. De telles mesures sont construites ici pour la première fois. Nous $n^{\prime}$ avons pas de point d'appui pour interpréter ces premiers résultats. Nous avons surtout le réflexe de questionner nos instruments de mesure. Mais si on s'y fie le moindrement, ces instruments nous disent que nos efforts les plus urgents pour accroitre la force d'attraction de nos ressources touristiques devraient porter sur la sacralisation on sight plutôt que sur les guides touristiques.

Par exemple, les attractions suivantes ont reçu le score $A$ dans les guides et le score D sur le terrain $(\mathrm{D}=$ score le plus bas $=$ absence totale de markers).

- Le fleuve Saint-Laurent

- Le panorame du haut du mont SteAnne

- La basilique Notre-Dame de Québec

- Le couvent des ursulines

- Le tour de l'île d'Orléans
On y trouve quelques-unes des ressources naturelles et culturelles hors-pair de la région de Québec. Dans les guides touristiques, on fait tout pour les rendre désirables à fréquenter ou à visiter. Mais, sur place, le touriste est laissé à lui-même pour tout découvrir.

La beauté du fleuve, quand on arrive sur la terrasse Dufferin, s'impose d'elle-même, direz-vous. Un écriteau précisant qu'il s'agit bien du St-Laurent ne servirait à rien. Pour un Québécois, le St-Laurent est intégré à sa culture depuis les bancs d'école. Mais pour le visiteur, qu'est-ce que c'est que ce fleuve? Alors, qu'on grave sur une pierre quelques vers de nos poètes à propos de cette voie fluviale, qu'une table d'orientation la situe bien dans l'espace; que des écriteaux en signalent les traits spécifiques, qu'un centre d'interprétation en rappelle l'histoire, la compare aux autres grands fleuves, etc. Alors, le St-Laurent, comme attraction à Québec, deviendra significative, donc inoubliable. Le touriste est tous yeux, toutes oreilles pour partager nos trésors, nos beautés; à nous cependant de faire les présentations. Á nous donc de faire que les promesses des guides touristiques ne soient pas que des leurres... f
(1) Editions du Cerf, Paris, $158 \mathrm{p}$.

(2) L'évaluation des attractions touristiques au Qub bec. Essai de móthodologie; par Marc Laplante, assisté de Louise Trottier, UOAM, Département d'Etudes urbaines et centre de recherche en gestion, Octobre 1984 (version préliminaire)

(3) La recherche a tút subventionnée par le ministère de l'Industrie, du Commerce et du Tourisme du Québec (il faut parler maintenant du ministảne du Tourisme du Quebecl. Le mandat portait explicitement sur l'élaboration de critêres objectifs pour etablir une classification hiérarchique des attractions touristiques. Le projet a commencé en mai 1984

(4) Vol. 2, no 2, juin 1983: "Regards neufs sur les aftracfions fouristiques au Quebec". Coordina. tion du numéro par Marc Laplante.

(5) Ceci est une partie de l'échantillon d'une quinzaine de guides touristiques que nous avions choisis a partir d'un examen critique d'une quarantaine de guides environ. N.B.: Le mode d'analyse de ce guide ainsi que leur choix seront révisés dans la version finale de la méthodologie.

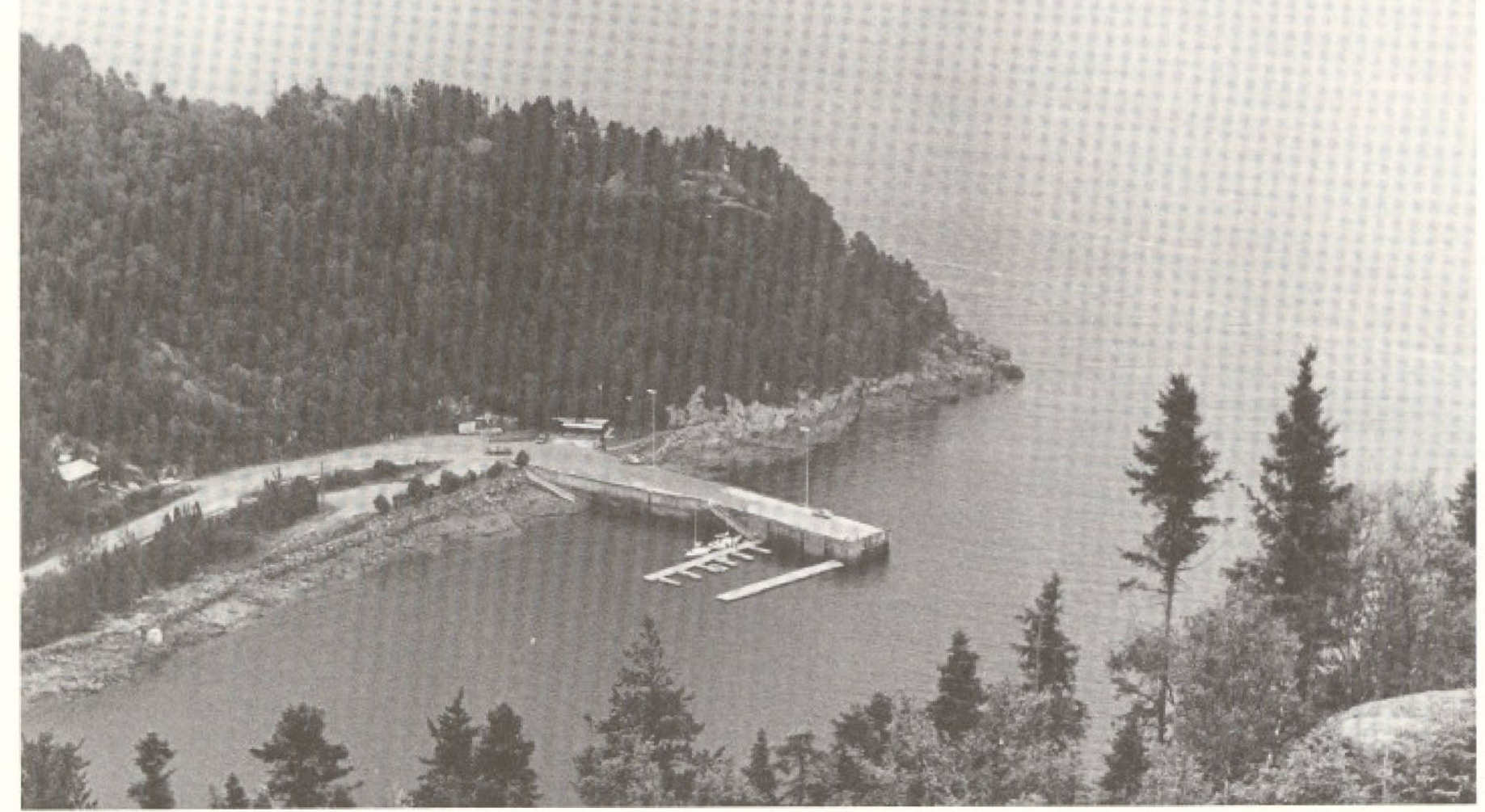

Les efforts les plus urgents pour accroïtre la force d'attraction de nos ressources touristiques devraient porter sur la sacralisation on sight plutôt que dans les guides touristiques. 